\title{
Modelling and verification of the electrical properties of organic dielectric monolayers in capacitive configurations
}

\author{
Michael B. Cortie $^{* a}$, Hadi Zareie ${ }^{\mathrm{a}}$, Jingquan Liu ${ }^{\mathrm{a}}$, Karl-Heinz Müller ${ }^{\mathrm{b}}$ and Michael J. Ford ${ }^{\mathrm{a}}$ \\ ${ }^{\text {a}}$ Institute for Nanoscale Technology, University of Technology Sydney, PO Box 123, Broadway \\ NSW 2007, Australia; \\ ${ }^{b}$ CSIRO, Industrial Physics, PO Box 218, Lindfield NSW 2070, Australia
}

\begin{abstract}
The possible role of self-assembled monolayers (SAMs) as the dielectric component of nanoscale capacitors is considered. SAMs of two rather different molecules, $\alpha, \alpha$ '-p-xylyldithiol ('XYL') and dodecanedithiol ('C12') were produced on a gold $\{111\}$ substrate, and characterized with respect to their conductivity. The data were fitted with a double tunnel barrier model, in which the two SAMs are primarily differentiated by barrier height and thickness with that of XYL having a thickness of $1.0 \mathrm{~nm}$ and a barrier height of $0.78 \mathrm{eV}$ compared to $1.69 \mathrm{~nm}$ and $1.39 \mathrm{eV}$ for $\mathrm{C} 12$. The remaining parameters of the model were determined by Monte Carlo optimization. Assuming perfect connection of top and bottom electrodes, the leakage current through the XYL at 1 volt is calculated to be $1.4 \times 10^{5} \mathrm{~A} / \mathrm{cm}^{2}$, compared to $2.7 \times 10^{4} \mathrm{~A} / \mathrm{cm}^{2}$ through $\mathrm{C} 12$. These values are not as low as can be obtained with $\mathrm{SiO}_{2}$ of the same thickness. However, SAMs are readily and precisely produced by simple, low temperature processes, a factor which may allow them a niche in the future.
\end{abstract}

Keywords: dielectric properties, self-assembled monolayers, organic electronics, electron tunneling

\section{INTRODUCTION}

Further miniaturization in electronics is now limited as much by electron tunneling through nanoscale oxide dielectric layers as by wavelength-imposed restrictions of lithography ${ }^{1}$. The dielectric in common use is $\mathrm{SiO}_{2}$, but much interest is currently directed towards investigating insulators with greater dielectric constants, such as $\mathrm{Al}_{2} \mathrm{O}_{3}{ }^{2}$, which could therefore be applied as thicker layers in capacitors. This strategy reduces the leakage current for a given capacitance density and device voltage. Here however we consider the concept of using self-assembled layers of organic molecules as the dielectric of a capacitor. The idea of using SAMs as the insulating layer in devices has been mooted before ${ }^{13}$ but to our knowledge this has not yet been considered explicitly in terms of capacitors. We note that use of a SAM as a dielectric layer in an electronic device is at present incompatible with the manufacturing techniques used in the electronics industry. However, a monolayer of organic molecules has the advantage that it can be self-assembled from a low-lemperature parent liquid, and that a tunnel barrier of precisely controllable thickness and barrier height can be produced $^{3}$. We believe that such layers have the potential to be developed as insulating or multifunctional dielectric layers in future nanoscale electronic circuits.

Electron conduction through SAMs is due to tunneling ${ }^{4}$, and depends sensitively upon the shape, height and width of the associated energy barrier. We present a semi-empirical model for a metal-SAM-metal system which is based on the wellknown WKB approximation, and show how the model can describe conduction through various types of monolayer configurations. Experimental data for a partially conjugated and a fully saturated molecule are presented and analyzed using the model. Reports in the prior literature claimed that the conductance of these two types of molecule would differ substantially, a point which we explore here.

"Michael.cortie@uts.edu.au, phone +61-2-9514-2208, fax +61-2-9514-7443, www.nano.edu.au 


\section{METHODOLOGY}

\subsection{Experimental}

Two molecules were used to make the SAMs, $\alpha, \alpha$ '-p-xylyldithiol (or 'XYL') and dodecanedithiol (designated here as 'C12'). The molecules are illustrated in Fig. 1. The molecules form SAMs of different thicknesses $1.0 \mathrm{~nm}$ vs $1.7 \mathrm{~nm}$ respectively once the $\mathrm{Au}-\mathrm{S}$ bonds are taken into account ${ }^{5,6}$ and are different in terms of their backbone, with XYL possessing a mixture of single and double carbon-carbon bonds, while that of $\mathrm{C} 12$ contains 12 carbon-carbon single bonds.

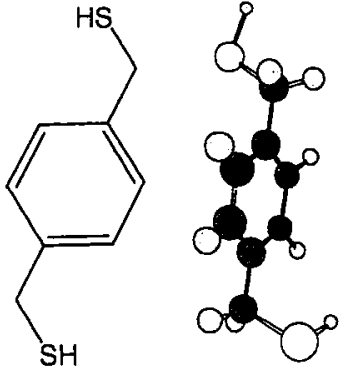

XYL

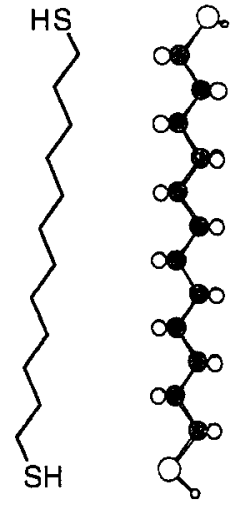

C12

Fig. I Structure of the two molecules studied in the present work.

The SAM of XYL was made by immersing freshly cleaned gold $\{111\}$ surfaces in a $0.5 \mathrm{mM}$ solution of $\mathrm{XYL}$ in methylene chloride for 24 hours. The SAM of C12 was similarly produced by immersing a gold $\{111\}$ surface in a $1 \mathrm{mM}$ solution of $\mathrm{C} 12$ in ethanol for 24 hours. In each case a gold nanoparticle top electrode was subsequently introduced onto the surface by dipping the SAM-coated samples in a colloidal suspension of $5 \mathrm{~nm}$ diameter citrate-stabilized gold nanoparticles for 24 hours. Scanning tunneling spectroscopy (STS) was performed on an Easyscan ${ }^{\text {TM }}$ system, but images of the SAM were obtained with a Multimode ${ }^{\mathrm{TM}}$ system, both operated in air at room temperature. Freshly cut Pt-Ir tips $(0.2 \mathrm{~mm})$ were used. STM images and STS curves were obtained with typical tip-sample bias voltages of up to $\pm 2 \mathrm{~V}$ and tunneling currents up to $100 \mathrm{nA}$.

HOMO-LUMO gaps were calculated using the Gaussian03 package ${ }^{7}$ within density functional theory (DFT) using a double zeta basis set with a single polarisation function. More specifically the B3LYP ${ }^{8}$ functional was employed together with a $6-31 G(d)$ basis set. This level of calculation is known to produce reasonable optimum geometries. HOMO-LUMO gaps were calculated for minimum energy structures. A number of points should be borne in mind when comparing these calculated gaps with experiment. Strictly speaking, DFT does not provide single particle energies and it is well known that DFT band gaps do not compare well with experimental values. Furthermore, it must be remembered that the LUMO is a virtual orbital and therefore does not necessarily correspond to the energy the equivalent orbital would have if it where occupied.

\subsection{Analytical model for a double tunnel barrier}

The I-V curve across a SAM may be readily measured in an STM, but the resulting data is due to a combination of tunnelling through the SAM and through the gap between STM tip and SAM. A method of disentangling these two currents is useful since it allows the effects due to the SAM to be isolated. However, while diverse attempts have been made to compute the currents through a SAM $a b$ initio $^{9,10}$, success requires an extraordinarily accurate determination of 
diverse input parameters ${ }^{9}$. In practice, results computed by $a b$ initio approaches may be in error by one or two orders of magnitude ${ }^{10,11.12}$. An alternative approach based on the semi-empirical WKB model has therefore been frequently adopted $^{4.13}$. In this method system parameters are determined by fitting the model to experimental data.

We have shown elsewhere how the current across a SAM can be characterized using a double tunnel model [Cortie, submitted]. The system is illustrated in Fig. 2, which invokes a dielectric barrier and an air barrier. The shape of both barriers is modelled using

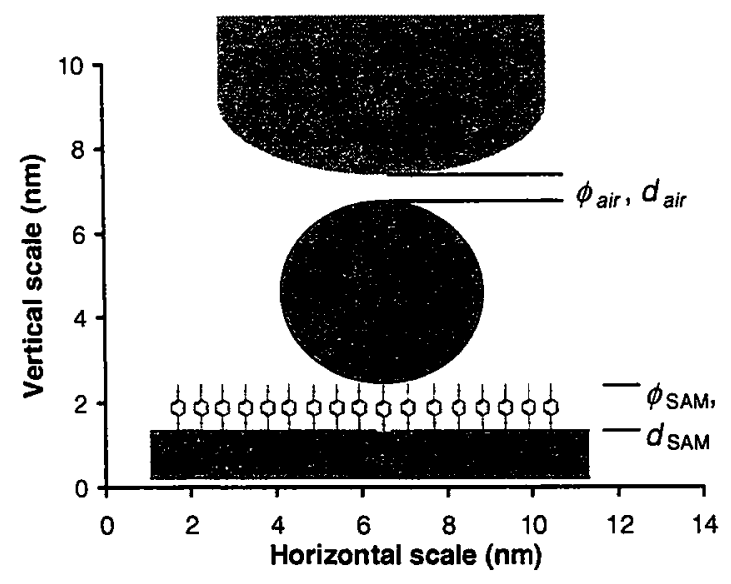

Fig 2. Schematic diagram showing arrangement of conductive substrate held at voltage $V_{\text {sub }}$, the SAM, a gold nanoparticle as top electrode which is at $V_{\text {part }}$ volts, and the tip of the STP, at $V_{\text {ip }}$ volts. Electrons must tunnel over both the air gap and the SAM to produce a current.

$\phi(z)=V_{s u b}\left(1-\frac{z}{a}\right)+\phi_{\max } \cdot\left[\sin \left(\frac{\pi \cdot z}{a}\right)\right]^{0.2}$

where $z$ is the distance along barrier of width $a$, and $\phi_{\max }$ is the maximum barrier height for either air or the SAM. Equ. (1) gives a more realistic barrier profile than that due to a simple trapezoid because it takes up the effect of image charges in an empirical way and bundles them into an effective value for the $\phi_{\max }$. The expressions for $\phi(z)$ may be used to generate an expression for tunnelling probability based on the WKB approximation ${ }^{14}$ :

$T(E)=e^{\left.\frac{2 \sqrt{2}}{\hbar} \int_{0}^{a} \sqrt{m((\phi(z)-E}\right) d z}$

where $E$ the energy of the incident electron and $m$ is its mass. We have performed the integration numerically, using the trapezoidal rule and a step size of $a / 100$. Although the 'effective mass' of the electron has been sometimes used ${ }^{4,15}$ in tunneling calculations, it would introduce an additional fitting parameter, and we have elected here to rather bundle this effect in with the effective barrier height. Having $T(E)$, and assuming a constant and equal density of states fully occupied up to identical Fermi levels in both substrate and tip ${ }^{16}$, and $V_{\text {tip }}=$ ground $=0 \mathrm{~V}$, then the net difference between the forward and reverse tunneling currents across the barrier is given by

$i=A^{\prime} \int^{V_{\text {sub }}} \rho_{\text {sub }}\left(E+e V_{\text {sub }}\right) \cdot \rho_{\text {tip }}(E) T(E) d E$

where $\rho_{\text {sub }}\left(E+e V_{\text {sub }}\right)$ is the density of states of the substrate after application of the bias voltage, and $A^{\prime}$ is a geometric parameter that takes into account the number of electrons available for tunnelling as a result of variations in crosssectional area and bulk electron density, and the dimensional requirements of (3). If we assume a simple and constant density of states up to the Fermi level then the expression for tunnelling current becomes 
$i=A \int_{0}^{V_{\text {sub }}} T(E) d E$

where the $A$ term includes the magnitude of the constant density of states. There is a unique voltage on the attached nanoparticle which will make the currents through the SAM and air equal, and we determine this for each $V_{\text {bias }}$ on the I-V curve using a iterative computer program. The computed composite I-V curve depends on the values of $\phi_{S \mathrm{AM}}, \phi_{\mathrm{air}}, d_{\mathrm{SAM}}$, $d_{\text {air }}, A_{\text {SAM }}$ and $A_{\text {air }}$, and the process is repeated in a Monte Carlo fashion with random changes to selected parameters to drive down the error of the fit. However, the model has too many unknowns for unambiguous application to a single I-V curve, and it is necessary to apply the known values for $d_{\mathrm{SAM}}\left(1.0 \mathrm{~nm}\right.$ for $\mathrm{XYL}^{5}$ and $1.69 \mathrm{~nm}$ for $\left.\mathrm{C} 12^{6}\right), \phi_{\mathrm{SAM}}(0.78 \mathrm{eV}$ for XYL [Cortie, submitted] and $1.39 \mathrm{eV}$ for $\mathrm{C} 12^{4}, \phi_{\mathrm{air}}\left(\sim 0.35 \mathrm{eV}^{17}\right)$ and a value for $A_{\text {air }}\left(\sim 4.5 \times 10^{20} \mathrm{nA}\right.$, determined for the Easyscan experimental set-up [Cortie, submitted]) to make the optimization manageable.

The values reported in the literature for $\phi_{\text {air }}$ vary between 0.1 to $0.95 \mathrm{eV}^{18}$, with some evidence that it is influenced by the microstructure and purity of the gold ${ }^{17}$ as well as by the ambient humidity ${ }^{18}$. These values are much less than the work function of $\mathrm{Au}$ in vacuum $(5.1 \mathrm{eV})$ due apparently to the effects of adsorbed contaminants ${ }^{17.19}$. However, the double tunnelling model is hardly sensitive to the value used for $\phi_{\mathrm{ar}}$, provided $d_{\text {air }}$ is small, because the voltage drop between STM tip and Au substrate is dominated by that across the SAM.

\section{RESULTS}

\subsection{Films}

Images of the monolayers produced in the present work are shown in Figure 3. It is known that these molecules are actually oriented at $24^{\circ 2}$ and $30^{\circ 21}$ respectively to the $\mathrm{Au}\{111\}$ surface, however this factor does not materially affect their electron conductivity ${ }^{1}$ and so is not considered further here.

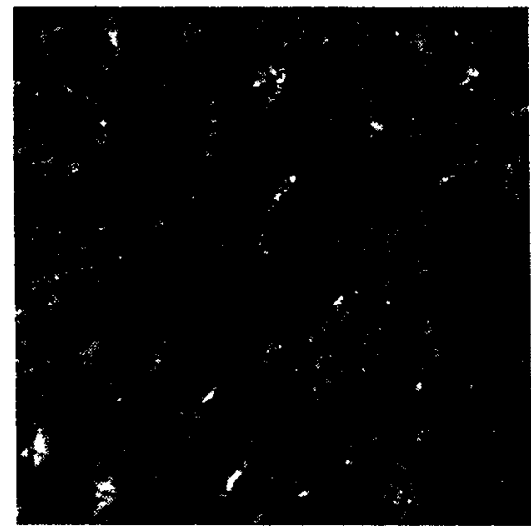

$50 \times 50 \mathrm{~nm}$

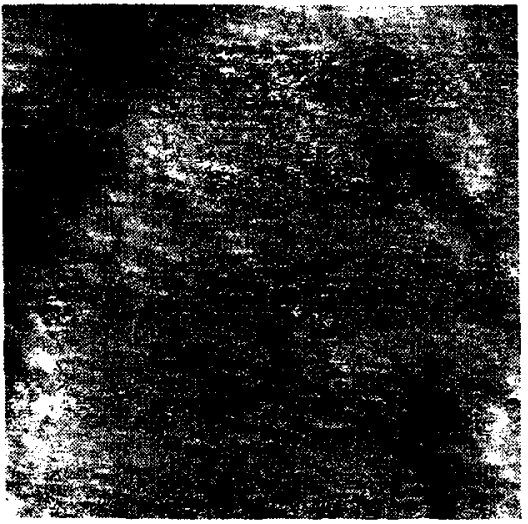

$19 \times 19 \mathrm{~nm}$

(a)

(b)

Fig.3 High resolution STM images of the SAMs prepared in the current work, (a) $\alpha, \alpha$-p-xylyldithiol, (b) dodecanedithiol.

\subsection{STS spectra}

STS scans taken at a selected nanoparticle on each SAM and with varying stand off distances, $d_{\text {air }}$, are shown in Figures 4 and 5. 


\subsection{Determination of remaining model parameters}

Once the parameters of the air gap have been determined, those still undetermined for the SAM can be found by a similar analysis of STS curves taken with different stand-off distances $\left(d_{\text {air }}\right)$. In the case of the SAM of XYL values for $A_{\mathrm{XYL}}$ and $\phi_{\mathrm{XY}}$ of $2 \times 10^{22} \mathrm{nA}$ and $0.78 \mathrm{eV}$ were obtained previously [Cortie, submitted], whereas for the SAM comprised of the $\mathrm{C} 12$ molecule best-fitting values of $A_{\mathrm{Cl} 2} \approx 5 \times 10^{24} \mathrm{nA}$ and $\phi_{\mathrm{Cl} 12} \approx \sim 1.4 \mathrm{eV}$ were indicated, Fig. 6 . The best-fitting value of $A_{\mathrm{Cl} 2}$ was determined by fitting the model to the data for the four data sets shown in Fig. 5 . Since $A_{\mathrm{C} 12}$ must have a common value for all four curves (because they were measured at the same position on the SAM), the best-fitting value of it is given by the maximum in the averaged values of correlation coefficient of all four data sets as shown in Fig. 6.

The calculated HOMO-LUMO gap for the XYL molecule was $5.7 \mathrm{eV}$ and that of the $\mathrm{C} 12$ was $7.1 \mathrm{eV}$, which is far greater than the effective barrier heights measured here. Two explanations may be offered for this. In the first instance the calculated values are for isolated molecules whereas the barrier heights apply to molecules that are chemically attached to a gold surface, a factor well-known to perturb their electronic configurations. Secondly, the Fermi level of the electrodes on either side of the molecule need not necessarily be midway between HOMO and LUMO, and in this case could be closer to the LUMO.

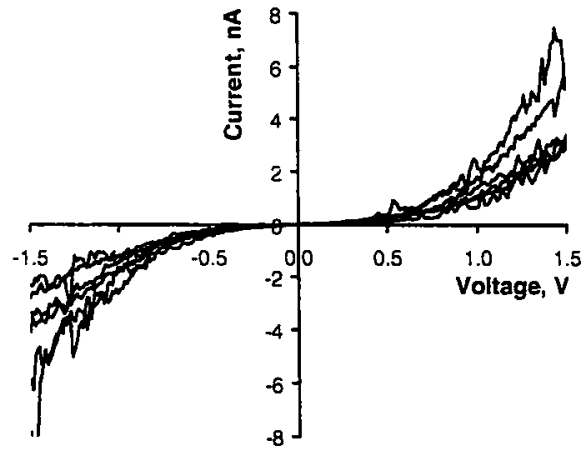

Fig. 4 Series of $\mathrm{I}-\mathrm{V}$ scans produced by scanning tunneling spectroscopy on a Au-XYL-Au-air-Pt tunnel barrier produced using varying stand off distances, $d_{\text {air }}$.

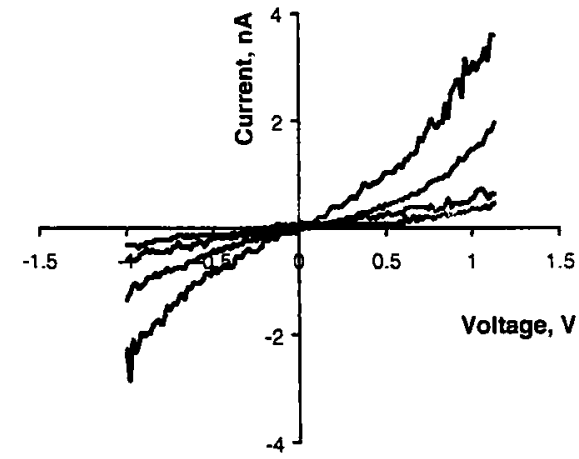

Fig. 5. Series of I-V scans produced by scanning tunneling spectroscopy on a Au-C12-Au-air-Pt tunnel barrier produced using varying stand-off distances, $d_{\text {iir }}$

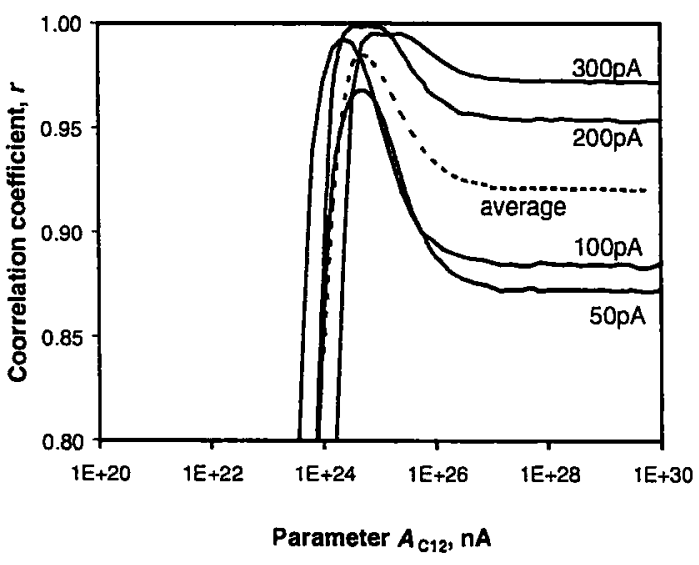

Fig 6. Correlation coefficient versus parameter $A_{\mathrm{C} 12}$ for different set currents. 


\section{DISCUSSION}

\subsection{Leakage currents}

Once the parameters of the double tunnel model have been estimated then the two tunnel barriers can be readily separated in subsequent analysis. Here we show the calculated leakage currents, expressed as $\mathrm{A} / \mathrm{cm}^{2}$ flowing through a 5 nm diameter gold nanoparticle attached to the top of a SAM. It is clear that the predicted leakage through the C12 SAM at 1 volt is about one fifth that through the XYL $\left(2.7 \times 10^{4} \mathrm{~A} / \mathrm{cm}^{2}\right.$ vs $\left.1.4 \times 10^{5} \mathrm{~A} / \mathrm{cm}^{2}\right)$. However, note that the SAM of C12 is about $60 \%$ thicker than that of the XYL, so all else being equal and assuming $\kappa=1.5$ for both, a nanoscale parallel plate capacitor device made using it as a dielectric will produce only $\sim 0.8 \mu \mathrm{F} / \mathrm{cm}^{2}$, compared to the $\sim 1.3 \mu \mathrm{F} / \mathrm{cm}^{2}$ available from the device using XYL as a dielectric. The 'gap resistance' of the junctions made with XYL and C12 is given by $\mathrm{d} V / \mathrm{d} I$ at $l=0$, after compensating for the effect of the air gap. In the present case, application of the fitted parameters to the model indicated a likely value of $1.2 \times 10^{8} \Omega$ for XYL and $8.6 \times 10^{8} \Omega$ for C12. In the case of $\mathrm{C} 12$ the value given is somewhat less than a published ${ }^{6}$ value of $4.1 \times 10^{9} \Omega$, but we consider the agreement to be acceptable for the present purposes, especially considering the large inter-laboratory scatter of STS data.

Leakage currents across SAM layers have also been recently measured in vacuum by Labonte ${ }^{1}$ who found that, at 1 volt. the current across a $2 \mathrm{~nm}$ thick SAM of resorcinarene $\mathrm{C} 10$ tetrasulfide was $\sim 10 \mathrm{~A} / \mathrm{cm}^{2}$, similar to that which would flow across $1.5 \mathrm{~nm}$ of $\mathrm{SiO}_{2}$ at that bias ${ }^{15}$. The current across 1.4 to $1.5 \mathrm{~nm}$ thick SAM of dodecanethiol was $\sim 1500 \mathrm{~A} / \mathrm{cm}^{2}$, similar to that across $1.0 \mathrm{~nm}$ thick $\mathrm{SiO}_{2}$ at the same voltage ${ }^{1}$. Note however, that the leakage current in $\mathrm{SiO}_{2}$ seems to be very sensitive to material condition, and others have reported leakage currents about an order of magnitude smaller ${ }^{22}$. In any event, the leakage current reported by Labonte across the C12 SAM in vacuum is an order of magnitude less than measured here in air. Evidently, the environment plays an important role in these systems. The barrier height in $\mathrm{SiO}_{2}$ has been given as 2.6 to $3.3 \mathrm{eV}^{15}$. This value is considerably higher than that of either of the two molecules examined here, and explains the excellent performance of $\mathrm{SiO}_{2}$ as a dielectric insulator.

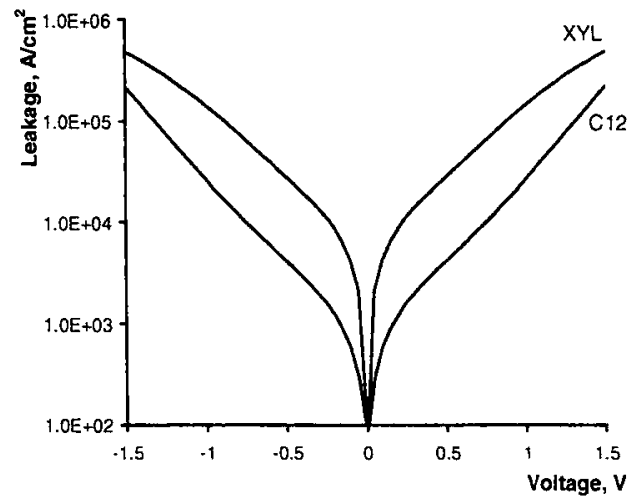

Fig. 7 Comparison of calculated leakage currents for SAMs comprised of XYL and C12.

\section{CONCLUSIONS}

The resistance of a dielectric barrier to current leakage can be characterized by its thickness and an effective barrier height. Self-assembled monolayers (SAMs) of organic molecules offer the possibility of precisely tailored dielectric layers for use in future electronic devices since their thickness and barrier height are readily manipulated. Here we have compared XYL, a partially conjugated species, with $\mathrm{C12}$, a fully saturated alkane. The XYL offers a lower barrier height in respective of electron tunneling and, together with its shorter length, this will cause leakage currents through it to be about five times larger than through $\mathrm{C} 12$ monolayers. However, nominal capacitances of $1.3 \mu \mathrm{F} / \mathrm{cm}^{2}$ will be possible using XYL compared to $0.8 \mu \mathrm{F} / \mathrm{cm}^{2}$ for $\mathrm{C} 12$. Neither is as good an insulator as $\mathrm{SiO}_{2}$, due primarily to having significantly lower barrier heights to electron tunneling in SAM systems than might be expected from their theoretical HOMO-LUMO gaps. 


\section{ACKNOWLEDGEMENTS}

Calculations were performed on the Australian Partnership for Advanced Computing (APAC) National Facility under a grant awarded through the Australian Centre for Advanced Computing and Communications (ac3) Partner Share scheme.

\section{REFERENCES}

1. A.P. Labonte, S.L. Tripp, R. Reifenberger and A. Wei, "Scanning Tunneling Spectroscopy of Insulating SelfAssembled Monolayers on Au(111)" J. Phys. Chem., 106, 8721-8725, 2002.

2. S. Meng, C. Basceri, B. W. Busch, G. Derderian, and G. Sandhu, "Leakage mechanisms and dielectric properties of $\mathrm{Al}_{2} \mathrm{O}_{3}$ 'TiN-based metal-insulator-metal capacitors", Applied Physics Letters, 83, 4439-4431, 2003.

3. T. Ohgi, H.Y. Sheng, and H. Nejoh, "Au particle deposition onto self-assembled monolayers of thiol and dithiol molecules", Applied Surface Science, 130-132, 919-924, 1998.

4. W. Wang, T. Lee and M. A. Reed, "Mechanism of electron conduction in self-assembled alkanethiol monolayer devices", Physical Review B, 68, 035416, 2003.

5. M. Dorogi, J. Gomez, R. Osifchin, R.P. Andres, and R. Reifenberger, "Room-temperature Coulomb-blockade from a self-assembled molecular nanostructure", Physical Review B, 52, 9071-9077, 1995.

6. A. S. Blum, J.C. Yang, R. Shashidhar, and B. Ratna, Comparing the conductivity of molecular wires with the scanning tunneling microscope, Applied Physics Letters, 82, 3322-3324, 2003.

7. M.J. Frisch et. al. Gaussian 03, Revision C.02, Gaussian, Inc., Wallingford CT, 2004.

8. A.D. Becke, J. Chem. Phys. 98, 5648, 1993.

9. L.E. Hall, J.R. Reimers, N.S. Hush, and K. Silverbrook, "Formalism, analytical model, and a priori Green'sfunction-based calculations of the current-voltage characteristics of molecular wires", Journal of Chemical Physics, 112, 1510-1521, 2000.

10. A. Nitzan, and R.A. Ratner, "Electron transport in molecular wire junctions", Science, 300, 1384-1389, 2003.

11. S.T. Pantelides, M. Di Ventra, N.D. Lang, and S.N. Rashkeev, "Molecular Electronics by the Numbers", IEEE Transactions on Nanotechnology, 1, 86-90, 2002.

12. K. Stokbro, J. Taylor, M. Brandbyge, J.L. Mozos, and P. Ordejón, "Theoretical study of the nonlinear conductance of di-thiol benzene coupled to Au( $\left(\begin{array}{llll}1 & 1 & 1\end{array}\right)$ surfaces via thiol and thiolate bonds", Computational Materials Science, 27 151-160, 2003.

13. A.L. Korotkov, M. Bowman, H.J. McGuinness, and D. Davidovic, "Influence of $\mathrm{H}_{2} \mathrm{O}$ molecules on sub-nanometre scale gaps between Au leads", Nanotechnology, 14, 42-45, 2003.

14. J. Singh, Modern Physics for Engineers, Wiley:New York, 1999.

15. F. Jiménez-Molinos, F. Gámiz, A. Palma, P. Cartujo, and J.A. López-Villanueva, "Direct and trap-assisted elastic tunneling through ultrathin gate oxides", Journal of Applied Physics, 91, 5116-5124, 2002.

16. A.I. Onipko, K. Berggren, F. Yu, O. Klymenko, L.L Malysheva, J.J.W. Rosink, L.J. Geerligs, E. van der Drift, and S. Radelaar, "Scanning tunneling spectroscopy on p-conjugated phenyl-based oligomers: A simple physical model", Physical Review B, 61, 11118-11124, 2000.

17. L. Boyer, S. Noël, and F. Houzé, "Apparent tunnel barrier heights of PtIr-Au interfaces in relation to the Au surface composition", J. Vac. Sci. Technol. B, 16, 2006-2012, 1998.

18. M-B. Song, J-M. Jang, S-E Bae, and C-W. Lee, "Charge transfer through thin layers of water investigated by STM, AFM and QCM", Langmuir, 18, 2780-2784, 2002.

19. G. Seine, R. Coratger, A. Carladous, F. Ajustron, R. Pechou, and J. Beauvillain, "Tip-to-surface distance variations vs voltage in scanning tunneling microscopy", Physical Review B, 60 11045-11050, 1999.

20. R.P. Andres, S. Datta, D.B. Janes, C.P. Kubiak, and R. Reifenberger, "The design, fabrication, and electronic properties of self-assembled molecular nanostructures", in Handbook of Nanostructured Materials and Nanotechnology, H.S. Nalwa Ed., Orlando: Academic Press, 179-231, 2000.

21. K. Kobayashi, T. Horiuchi, H. Yamada and K. Matsushige, "STM studies on nanoscopic structures and electric characteristics of alkanethiol and alkanedithiol self-assembled monolayers", Thin Solid Films 331, 210-215, 1998.

22. C. Leroux, P. Mur, N. Rochat, D. Rouchon, R. Truche, G. Reimbold and G. Ghibaudo, Characterization and modeling of nanometric $\mathrm{SiO}_{2}$ dielectrics, Microelectronic Engineering 72, 121-124, 2004. 